\title{
Influence of friction stir process parameters on surface quality of aluminum alloy A2017
}

\author{
Cecile Langlade ${ }^{1}$, Adriana Roman ${ }^{1}$, Daniel Schlegel ${ }^{1,2}$, Eric Gete ${ }^{1}$, Patrice Noel $^{1}$, and \\ Milena Folea ${ }^{3, *}$ \\ ${ }^{1}$ Univ. Bourgogne Franche Comté, IRTES-M3M EA7274, 90010 Belfort Cedex, France \\ ${ }^{2}$ ESTA, 90000 Belfort Cedex, France \\ ${ }^{3}$ Transilvania University of Brasov, Department of Manufacturing Engineering, Mihai Viteazu No.5, \\ Braşov, Romania
}

\begin{abstract}
Friction stir processing is an environment friendly surface engineering process that enhances mechanical properties and refines microstructure of processed zones. The aim of this paper is to study the influence of process parameters during friction stir processing of aluminium alloy A2017 plates on surface quality of the processed zones.
\end{abstract}

\section{Introduction}

Friction stir, originally designed as a welding method, recently became used as a surface engineering method that allows obtaining in-situ ultrafine grains in surface layers. Friction stir processing (FSP) is a thermo-mechanical technique with a very simple basic principle [1]: a rotating tool is moved along the workpiece surface while applying an axial load to increase generated heat (Fig. 1).

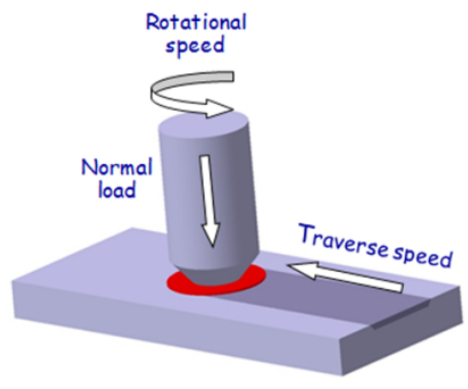

Fig. 1. Basic principle of friction stir processing [2].

Friction stir welding was patented in 1991 by British scientists of The Welding Institute (TWI) as an assembly method, but lately it has also been developed as a surface modification method due to its numerous technical, economic and environmental advantages $[1,3]$.

\footnotetext{
*Corresponding author: m.folea@unitbv.ro
} 
The similarity of friction stir process kinematics with milling process kinematics makes possible the use of conventional milling machines equipped with force control devices. This is one of major advantages of friction stir processing, because it allows finishing the surface and, in the meantime, it applies in-situ localized heat treatment leading to microstructural refinement, densification, homogeneity, increased microhardness, superior wear and corrosion resistance of the surface layer. Friction stir was initially applied to aluminium alloys [1] and extended lately to other materials such as copper alloys [4, 5], magnesium alloys [6,7], steels [8,9] or titanium alloys [10, 11].

Until now, aluminum alloys are by far the most widely used materials in FSP industrial applications; therefore numerous studies have been dedicated to the effect of FSP conditions on the processed zone. Karthikeyan et al. [12-14] studied the friction stir behavior of the aluminum alloys A2285, A413 and A319 in order to examine effect of feed rate and rotational speed on microstructure and properties. They reported increased tensile, yield strengths and ductility along with grain size reduction due to FSP. Pasebani et al. [15] studied the effect of tool rotation rate on microstructure of friction stir processed 2024Al alloy and found significant change in size and morphology of the constituent particles after FSP. After studying the effect of friction stir processing conditions on A356-T6 cast aluminum alloy, Tajiri et al. concluded that lower rotational speed led to better results enhancing fatigue resistance and eliminating casting defects [16].

Since all the available studies on FSP are focused on microscopic changes, mechanical and tribological properties enhancement induced by FSP, the purpose of this paper is to extend research of FSP and study the effect of process parameters on surface roughness.

\section{Experimental details}

\subsection{Materials and FSP equipment}

Workpiece material used in present study was aluminium alloy A2017, an alloy with high strength and good machinability after heat treatment, generally used for structural components, airspace industry and other industrial applications. This alloy is also known as $\mathrm{AlCu} 4 \mathrm{MgSi}$ and has the chemical composition presented in Table 1.

Table 1. Chemical composition of A2017 aluminium alloy (\%wt).

\begin{tabular}{|c|c|c|c|c|c|c|c|c|c|c|}
\hline $\mathbf{A l}$ & $\mathbf{S i}$ & $\mathbf{F e}$ & $\mathbf{C u}$ & $\mathbf{M n}$ & $\mathbf{M g}$ & $\mathbf{C r}$ & $\mathbf{Z n}$ & $\mathbf{T i}$ & $\begin{array}{c}\text { Other } \\
\text { each }\end{array}$ & $\begin{array}{c}\text { Other } \\
\text { total }\end{array}$ \\
\hline Bal. & $\begin{array}{c}0.20- \\
0.8\end{array}$ & $\begin{array}{c}0.7 \\
\max \end{array}$ & $\begin{array}{c}3.5- \\
4.5\end{array}$ & $\begin{array}{c}0.40- \\
1.0\end{array}$ & $\begin{array}{c}0.40- \\
0.8\end{array}$ & $\begin{array}{c}0.10 \\
\max \end{array}$ & $\begin{array}{c}0.25 \\
\max \end{array}$ & $\begin{array}{c}0.15 \\
\max \end{array}$ & $\begin{array}{c}0.05 \\
\max \end{array}$ & $\begin{array}{c}0.15 \\
\max \end{array}$ \\
\hline
\end{tabular}

Friction stir tests were performed on 100x30x8 (mm) rectangular plates with $8 \mathrm{~mm}$ thickness on a CNC Gambin milling center (Fig. 2a). One of the major advantages of friction stir processing is that in case of horizontal plane surfaces it can be done on vertical milling machines. Due to temperature rise during friction stir processing, normal force varies unless special devices are added to the milling machine. For this study the milling center was equipped with a pneumatic tool holder for maintaining constant the normal force (Fig. 2b).

In order to minimize tool wear, an alumina tool was chosen for the tests. Traditionally, friction stir tools have a pin (probe) and a shoulder [1]. Considering the brittleness of alumina and the fact that for the present study it was intended to modify only a thin surface layer of the samples, a pinless cylindrical tool with $10 \mathrm{~mm}$ diameter and $0.5 \mathrm{~mm}$ chamfer was selected. 


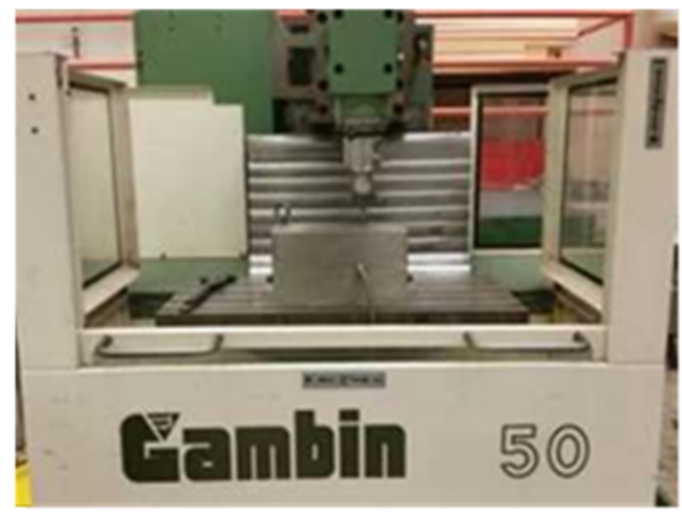

a)

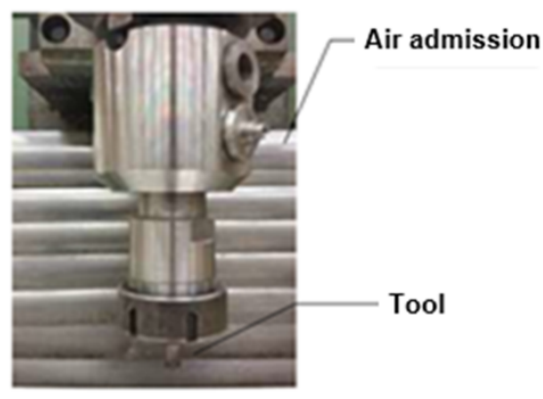

b)

Fig. 2. Experimental stand (a) with pneumatic toolholder (b).

Samples were visually inspected after FSP in order to evaluate the surface after processing, especially the marks left by the tool. Also, surface roughness was measured using a 3D laser profilometer.

\subsection{Process parameters}

Three parameters were taken into account as input variables: rotation speed (rpm), traverse speed $(\mathrm{mm} / \mathrm{min})$ and normal force $(\mathrm{N})$. Two variation levels were chosen for the rotation speed and the traverse speed, while for the normal force a third variation level was added considering the effect of this parameter on the deformation of the surface layer. The experiment design resulted had 12 runs $(2 \times 2 \times 3)$ and the parameter variation values were set as shown in table 2 .

Table 2. Experiment design.

\begin{tabular}{|l|c|}
\hline \multicolumn{1}{|c|}{ Parameter } & Test values \\
\hline \multirow{2}{*}{ Rotation speed (rpm) } & 300 \\
\cline { 2 - 2 } & 900 \\
\hline \multirow{2}{*}{ Traverse speed (m/min) } & 50 \\
\cline { 2 - 2 } & 100 \\
\hline \multirow{3}{*}{ Normal force (N) } & 500 \\
\cline { 2 - 2 } & 1000 \\
\cline { 2 - 2 } & 1500 \\
\hline
\end{tabular}

\section{Results and discussion}

The visual inspection of the samples after the tests revealed that for aluminium alloy A2017 the surface quality was highly dependent on the process parameters. In Fig. 3 are shown the extreme cases of the best two samples and most deteriorated two samples obtained after test runs with various parameters. 


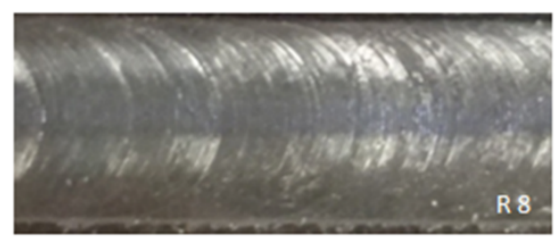

a)

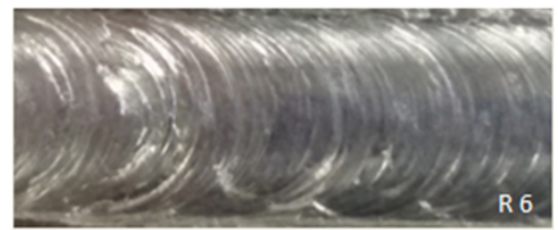

c)

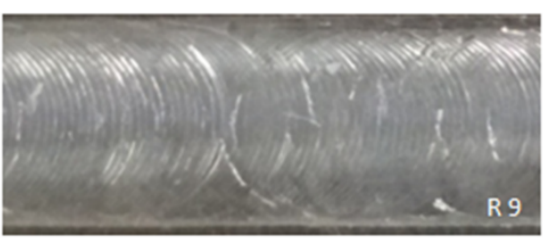

b)

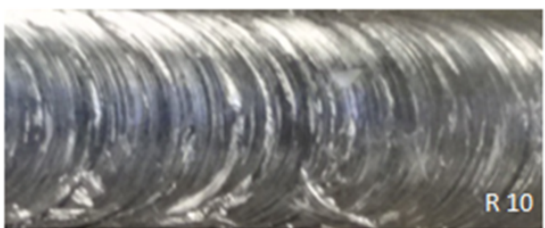

d)

Fig. 3. Visual comparison of friction stired zones with good surface appearance (a and b) and with deteriorated surface (c and d)

For a more accurate evaluation of the surface quality each sample was studied using laser profilometer as illustrated in Fig. 4.
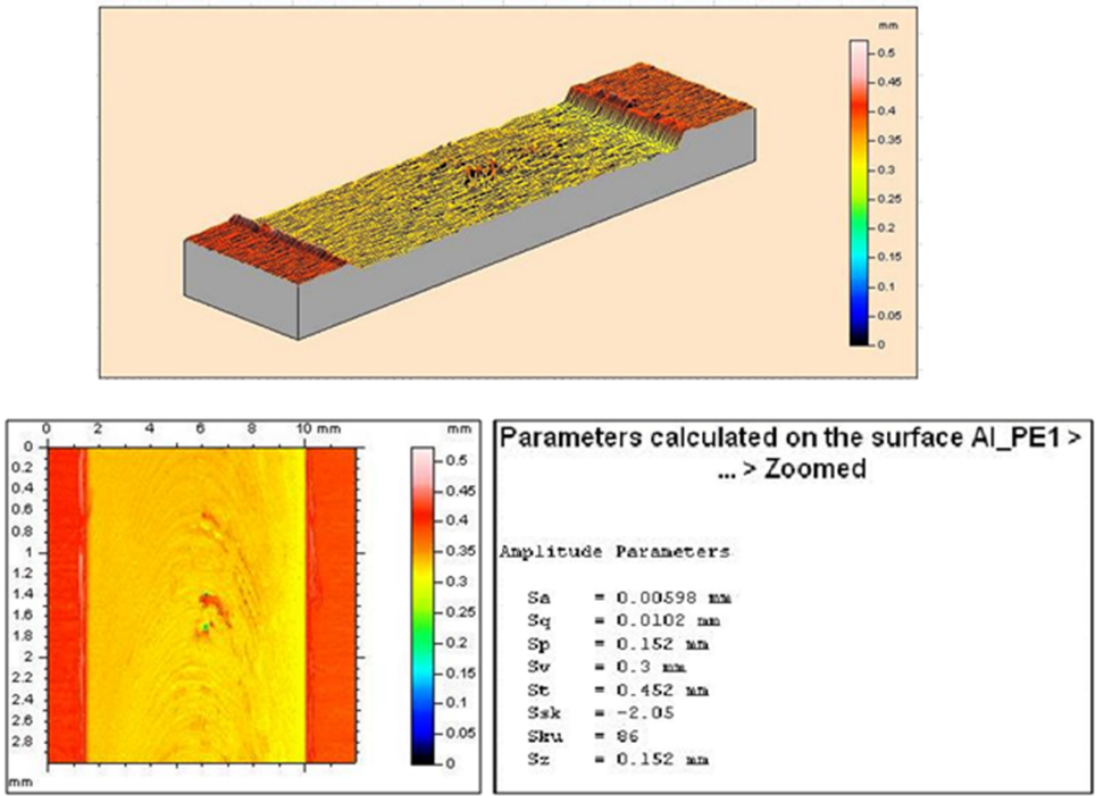

Fig. 4. Profilometric analysis for sample no.1 (50 mm/min, 500 N, $300 \mathrm{rpm})$.

To determine the influence of process parameters on the surface roughness after FSP, the surface roughness parameter $S_{a}$ was used. $S_{a}$ is average surface roughness and is evaluated over a $3 \mathrm{D}$ surface with the following formula:

$$
S_{a}=\iint_{a}|Z(x, y)| d x d y
$$

where $x$ and $y$ are 2D coordinates of points of the surface $a$ and $Z$ is height of the surface relative to the best fitting plane. 
Table 3. Surface roughness after FSP.

\begin{tabular}{|c|c|c|c|c|}
\hline $\begin{array}{c}\text { Test } \\
\text { run no. }\end{array}$ & $\begin{array}{c}\text { Traverse speed } \\
(\mathbf{m m} / \mathbf{m i n})\end{array}$ & Force $(\mathbf{N})$ & $\begin{array}{c}\text { Rotation speed } \\
(\mathbf{r p m})\end{array}$ & $\begin{array}{c}\text { Sa } \\
(\boldsymbol{\mu m})\end{array}$ \\
\hline 1 & 50 & 500 & 300 & 5.98 \\
\hline 2 & 50 & 500 & 900 & 12.5 \\
\hline 3 & 100 & 500 & 300 & 5.38 \\
\hline 4 & 100 & 500 & 900 & 8.73 \\
\hline 5 & 50 & 1000 & 300 & 8.36 \\
\hline 6 & 50 & 1000 & 900 & 17.4 \\
\hline 7 & 100 & 1000 & 300 & 8.73 \\
\hline 8 & 100 & 1000 & 900 & 2.82 \\
\hline 9 & 50 & 1500 & 300 & 3.62 \\
\hline 10 & 50 & 1500 & 900 & 24.1 \\
\hline 11 & 100 & 1500 & 300 & 7.92 \\
\hline 12 & 100 & 1500 & 900 & 14 \\
\hline
\end{tabular}

Surface roughness measurements are presented in table 3 and illustrated in Fig. 5. In both of them it can be observed that the best surface roughness with $S_{a}$ of $2.82 \mu \mathrm{m}$ was obtained for test run no. 8 performed at high level of traverse speed, medium level of normal force and high level of rotation speed. Tool traces in this case were quite uniform as shown in Fig. 3a. A good surface quality with a close value of surface roughness parameter $S_{a}(3.62 \mu \mathrm{m})$ was obtained for run no. 9 too, at lower traverse speed but at highest level of force and low level of rotation speed. In spite of the small rotation speed that reduced friction, the higher pressure of the tool and the lower traverse speed that maintained the heat longer in one spot decreased the surface quality, fact also revealed in Fig. 3 b. Very poor surface quality resulted in case of test runs no. 6 (Fig. 3 c) and no. 10 (Fig. 3d) with measured values for $S_{a}$ of $17.4 \mu \mathrm{m}$ and $24.1 \mu \mathrm{m}$, respectively. High rotation speed, medium and low normal force, corroborated with slow traverse movement made the tool to sink in the material and leave deep traces behind.

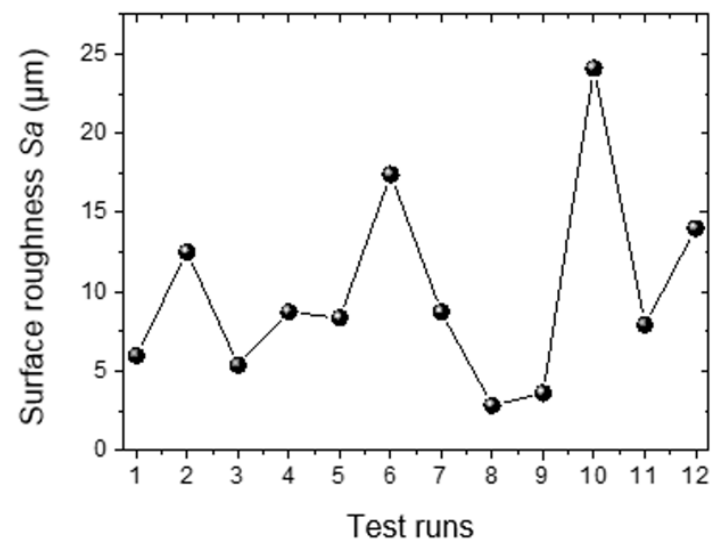

Fig. 5. Comparison of roughness $\left(S_{a}\right)$ 


\section{Conclusions}

This work aimed to determine the influence of process parameters during friction stir processing of aluminum alloy A2017 on surface quality of the processed zones.

The results obtained have shown that the optimal conditions in terms of roughness for FSP process applied to this material are represented by test conditions for runs $8(\mathrm{~F}=1000 \mathrm{~N}$, $\mathrm{f}=100 \mathrm{~mm} / \mathrm{min}, \mathrm{n}=900 \mathrm{rpm})$ and $9(\mathrm{~F}=1500 \mathrm{~N}, \mathrm{f}=50 \mathrm{~mm} / \mathrm{min}, \mathrm{n}=300 \mathrm{rpm})$ that provided the right combination between the amount of heat produced by normal force and rotation speed and the cooling rate determined by the traverse speed. Too much heat and low cooling rate seem to affect negatively the quality of the surface.

Further investigations have to be done on the influence of machining conditions, especially in what concerns the influence of FSP parameters on the stirred zone thickness and microstructure.

\section{References}

1. R.S. Mishra, .Z.Y. Ma, Mat. Sci. Eng. R 50, 1 (2005)

2. M. Folea, C. Langlade, A. Roman, D. Schlegel, E. Gete, D. Chamoret, Producing Nanograin Surface Layers by Friction Stir Processing - chapter in Comprehensive Guide for Nanocoatings Technology (Nova Science Publishers Inc., New York, 2015)

3. M. K. Kulekci, U. Esme, B. Buldum, Int. J. Adv. Manuf. Technol., 85(5), 1687 (2016)

4. S.K. Menon, F.A. Pierce, B.P. Rosemark, K. Oh-Ishi, S. Swaminathan, T.R. McNelley, Metall. Mater. Trans. A, 43, 3687 (2012)

5. K. Nakata, Weld. Int., 19(12), 929 (2005)

6. B.M. Darras, M.K. Khraisheh, F.K. Abu-Farha, M.A. Omar, J. Mater. Process. Technol., 191(1-3), 77 (2007)

7. D. Zhang, M. Suzuki, K. Murayama, Scripta Mater., 52(9), 899 (2005)

8. A. Chabok, K. Dehghani, Mat. Sci. Eng. A-Struct., 528(1), 309 (2010)

9. L. Wei, T. W. Nelson, Mat. Sci. Eng. A-Struct., 556, 51 (2012)

10. Y. Zhang, Y. S. Sato, H. Kokawa, S. H. C. Park, S. Hirano, Mater. Sci. Eng. A-Struct., 485(1-2), 448 (2008)

11. A. Lauro, Weld. Int., 26(1), 8 (2012)

12. L. Karthikeyan, , V.S. Senthilkumar, V. Balasubramanian, S. Natarajan, Mater. Design, 30(6), 2237 (2009)

13. L. Karthikeyan, , V.S. Senthilkumar, V. Balasubramanian, S. Arul, Mater. Lett., 64(3), $301(2010)$

14. L. Karthikeyan, , V.S. Senthilkumar, K.A. Padmanabhan, Mater. Design, 31(2), 761 (2010)

15. S. Pasebani, I. Charit, R.S. Mishra, Rajiv (2015). Mater. Lett. , 160, 64 (2015)

16. A. Tajiri, Y. Uematsu, T. Kakiuchi, Y. Tozaki, Y. Suzuki, A. Arfinaldi, Int. J. Fatigue, 80, $192(2015)$ 